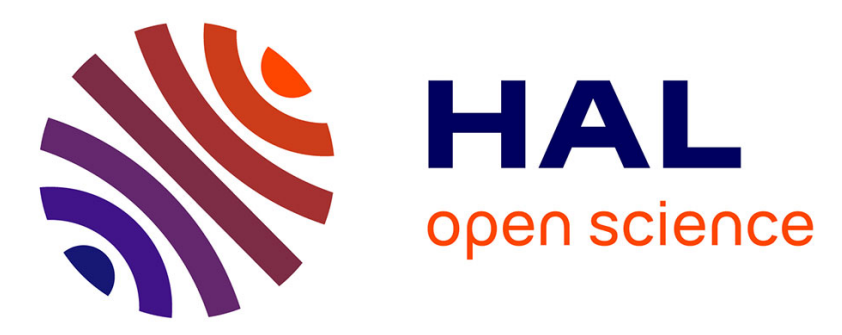

\title{
Effects of deposition temperature and elemental layer thickness on the properties of Fe/Co multilayers grown by molecular beam epitaxy \\ M Carbucicchio, R Ciprian, L Nasi
}

\section{- To cite this version:}

M Carbucicchio, R Ciprian, L Nasi. Effects of deposition temperature and elemental layer thickness on the properties of $\mathrm{Fe} / \mathrm{Co}$ multilayers grown by molecular beam epitaxy. Journal of Physics D: Applied Physics, 2010, 43 (40), pp.405001. 10.1088/0022-3727/43/40/405001 . hal-00569721

HAL Id: hal-00569721

https://hal.science/hal-00569721

Submitted on 25 Feb 2011

HAL is a multi-disciplinary open access archive for the deposit and dissemination of scientific research documents, whether they are published or not. The documents may come from teaching and research institutions in France or abroad, or from public or private research centers.
L'archive ouverte pluridisciplinaire HAL, est destinée au dépôt et à la diffusion de documents scientifiques de niveau recherche, publiés ou non, émanant des établissements d'enseignement et de recherche français ou étrangers, des laboratoires publics ou privés. 


\title{
Effects of deposition temperature and elemental layer thickness on the properties of $\mathrm{Fe} / \mathrm{Co}$ multilayers grown by molecular beam epitaxy.
}

\author{
M Carbucicchio ${ }^{1}$, R Ciprian $^{1}$ and L Nasi ${ }^{2}$ \\ ${ }^{1}$ Department of Physics, University of Parma, 43100 Parma, Italy \\ ${ }^{2}$ CNR-IMEM Institute, Parco Area delle Scienze 37/A, 43010 Fontanini-Parma, Italy \\ E-mail: massimo.carbucicchio@ fis.unipr.it
}

\begin{abstract}
Fe/Co multilayers were grown at different temperatures up to $200^{\circ} \mathrm{C}$ onto $\mathrm{MgO}-(100)$ monocrystalline substrates, varying both the Co and Fe elemental layer thickness. All samples show a multilayered structure where the elemental layers are separated by a thin interfacial region which, for high-temperature growths, is constituted by an equiatomic FeCo compound. The multilayers grown at room temperature show an in-plane uniaxial magnetic anisotropy. The increase of the growing temperature determines an improvement of the sample smoothness and the appearance of two preferred in-plane orientations of the easy magnetization axis having different strength. By increasing the Co layer thickness, the in-plane magnetic behaviour becomes isotropic, while by increasing the Fe layer thickness, an out-of-plane contribution to the magnetization vector establishes which is responsible for the appearance of a well defined magnetic morphology constituted by stripe-like domains.
\end{abstract}

\section{Introduction}

The great interest addressed to Fe/Co multilayers is due to their unique magnetic properties which found applications in the magnetic recording and the development of giant and tunneling magnetoresistance systems. These properties are strongly influenced by the microstructure and can be finely tailored adjusting the growing parameters. In particular both coercivity and anisotropy can be tailored by changing the film thickness, the order deposition of the elemental layers [1] and the degree of interface roughness [2] and crystallinity [3-5]. These last characteristics in turn, are largely depending on the nature and crystallinity of the substrates [1, 6] and on the deposition temperature, which can determine the establishing of peculiar crystallographic textures [6-9]. While many works have been carried out on Fe/Co films grown at room temperature, low attention has been addressed to the effects that the growing temperature plays on the microstructural and magnetic properties of $\mathrm{Fe} / \mathrm{Co}$ multilayers.

In the present work, thin $\mathrm{Fe} / \mathrm{Co}$ multilayers were deposited by molecular beam epitaxy (MBE) at different temperatures onto $\mathrm{MgO}-(100)$ monocrystalline substrates, changing the $\mathrm{Fe}$ 
and Co layer thickness. The magnetic and microstructural properties were studied as a function of both the growing temperature and the elemental layer thickness.

The increase of the growing temperature leaves almost unchanged the coercivity of the multilayers while appreciably influences the in-plane uniaxial anisotropy and the $\mathrm{Fe} / \mathrm{Co}$ interface intermixing. On the other hand, the elemental layer thickness influences the anisotropy dispersion along the easy magnetization axis of the multilayers grown at room temperature. For higher growing temperatures, the increase of the elemental layer thickness appreciably modifies the magnetic properties giving rise to an isotropic in-plane behaviour and to a perpendicular contribution to the magnetization vector.

\section{Experimental}

$\mathrm{Co} / \mathrm{Fe}$ multilayers, $60 \mathrm{~nm}$ thick, were grown in ultra-high vacuum onto $\mathrm{MgO}-(100)$ monocrystalline substrates by means of a MBE system equipped with electron guns, at different temperatures ranging from room temperature (RT) up to $200^{\circ} \mathrm{C}$. The deposition rates were for both elements $\sim 0.6 \mathrm{~nm} / \mathrm{min}$, and the layer thickness was controlled "in-situ" by means of a quartz microbalance. All multilayers were grown starting from Co and capped with an overlayer of Co $3 \mathrm{~nm}$ thick. To study the effects of the Co and Fe layer thickness on the magnetic properties of the multilayers, two series of samples were grown. In the first series the Fe layer thickness was fixed to $9 \mathrm{~nm}$ while the Co layer thickness $\left(t_{C o}\right)$ was changed in the range $3 \div 11$ $\mathrm{nm}$. In the second series the Co layer thickness was $3 \mathrm{~nm}$ and the Fe thickness $\left(t_{F e}\right)$ was changed in the range $9 \div 17 \mathrm{~nm}$. In the following the two series of samples were labelled as $\mathrm{T}$ $\mathrm{N}\left[\mathrm{Co}\left(t_{C o}\right) / \mathrm{Fe}\left(t_{F e}\right)\right]$ where $\mathrm{T}$ is the growing temperature in ${ }^{\circ} \mathrm{C}$ and $\mathrm{N}$ the $\mathrm{Co} / \mathrm{Fe}$ bilayer repetition number.

Conversion Electron Mössbauer Spectroscopy (CEMS) was performed by means of a 50 $\mathrm{mCi}{ }^{57} \mathrm{Co}(\mathrm{Rh})$ source, with the $\gamma$-rays at an incident angle of $15^{\circ}$. The isomer shifts are referred to $\alpha$-Fe. The spectra were fitted with a superposition of Lorentzian lines by means of a leastsquares minimization routine with a combination of linear and non-linear regressions.

The X-ray diffraction (XRD) measurements were performed in $\theta-2 \theta$ geometry by means of a Philips X'Pert diffractometer using the $\mathrm{Cu}(\mathrm{K} \alpha)$ radiation $(\lambda=0.154 \mathrm{~nm})$.

The magnetic measurements were carried out with a magneto-optical Kerr effect (MOKE) magnetometer using a s-polarized $633 \mathrm{~nm} \mathrm{He}-\mathrm{Ne}$ laser light and the modulation-polarization technique. The angular dependence of the normalized remanence, $M_{r} / M_{s}(\alpha)$, was studied applying the magnetic field parallel to the film plane at different angles, $\alpha . M_{r}$ is the remanence recorded for zero applied field and $M_{s}$ is the saturation magnetization. The magnetic interactions among ferromagnetic grains and layers were studied by means of the $\Delta M$-plot, where $\Delta M(H)$ is defined as:

$$
\Delta M(H)=M_{D C D}(H)-\left[1-2 M_{I R M}(H)\right] .
$$

$M_{I R M}(H)$ and $M_{D C D}(H)$ are respectively the isothermal and dc demagnetized remanence curves normalized to the saturation magnetization, and are obtained by plotting the remanence as a function of an increasing positive/negative magnetic field applied to a previously demagnetized/saturated samples. For a non-interacting assembly of particles $\Delta M$ is zero for any values of $H$. When exchange-coupling or demagnetizing interactions establish among the grains, $\Delta M$ shows positive or negative values respectively.

Conventional and high resolution transmission electron microscopy (HRTEM), high angle annular dark field (HAADF) and energy dispersive X-ray spectroscopy (EDS) in scanning mode (STEM) were performed on the 200-3[Co(11)/Fe(9)] and 200-3[Co(3)/Fe(17)] multilayers using a JEOL 2200FS microscope working at $200 \mathrm{keV}$. The STEM-HAADF and selective area EDS 
analyses provide atomic number (Z)-dependent contrast images, thus revealing the overall quality of the multilayers. Cross-section specimens were prepared by mechanical grinding and dimpling, followed by Ar ion milling in a Gatan PIPS apparatus.

Atomic and Magnetic Force Microscopy (AFM, MFM) were performed in ultra-high vacuum in tapping mode. In particular, MFM images were obtained with a CoCr-coated tip probe, in lift mode, at a distance of $40 \mathrm{~nm}$ from the sample surface. The values of the surface roughness were calculated from AFM images as the average deviation of the roughness profile from a mean line over the evaluation length. The sensitivity of AFM allows measuring the surface roughness over a very wide dynamic range from $0.03 \mathrm{~nm}$ to $500 \mathrm{~nm}$.

\section{Microstructural Properties}

The CEMS spectrum measured for the RT-5[Co(3)/Fe(9)] multilayer, figure $1(\mathrm{a})$, can be fitted as the superposition of three contributions: a main sextet $(\alpha)$ due to $\alpha-\mathrm{Fe}$, and two series of sextets $\left(\zeta\right.$ and $\chi$ ), whose hyperfine magnetic field $\left(\mathrm{H}_{\mathrm{hf}}\right)$ distributions are reported beside the spectrum. These contributions can be attributed respectively to a FeCo alloy having an almost equiatomic composition, and to a solid solution of $\mathrm{Fe}$ into $\mathrm{Co}, \mathrm{Co}(\mathrm{Fe})_{\mathrm{ss}}$ [6]. The corresponding XRD pattern reported in figure 2(a) shows only the (200) reflection due to the $\mathrm{MgO}$ substrate.

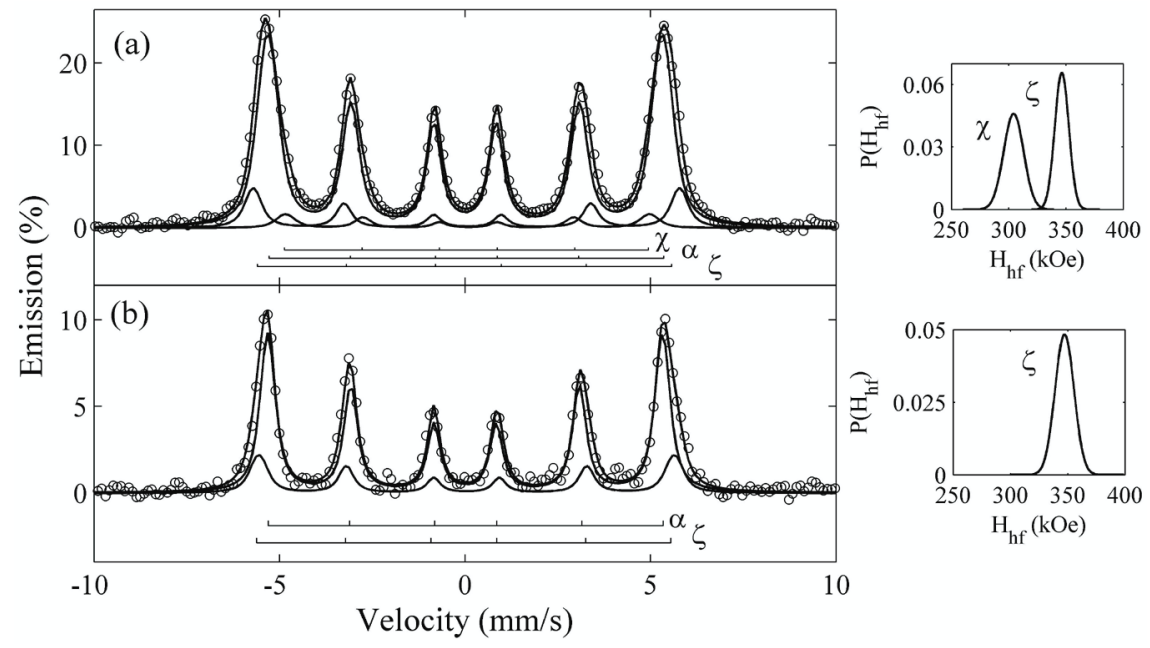

Figure 1 CEMS spectra for the (a) RT-5[Co(3)/ $\mathrm{Fe}(9)]$ and (b) $200-5[\mathrm{Co}(3) / \mathrm{Fe}(9)]$ multilayers. The hyperfine field distributions for the $\chi$ and $\zeta$ contributions are reported beside the spectra.

The increase of the growing temperature up to $200^{\circ} \mathrm{C}$, contrary to what normally expected, determines a lower intermixing at the interfaces. From the analysis of the relative areas of the contributions to the Mössbauer spectrum performed for the $100-5[\mathrm{Co}(3) / \mathrm{Fe}(9)]$ multilayer a decrease of the amount of the $\mathrm{Co}(\mathrm{Fe})_{\mathrm{ss}}$ can be deduced, followed by a slight increase of the $\alpha$-Fe amount. For the film grown at $200^{\circ} \mathrm{C}$, the Mössbauer spectrum, figure 1(b), shows the presence of $\alpha-\mathrm{Fe}$ and $\mathrm{CoFe}$, while the contribution from $\mathrm{Co}(\mathrm{Fe})_{\mathrm{ss}}$ has completely disappeared. This fact can be explained considering the higher atomic mobility which favours an elemental "layer-bylayer" growth, this way lowering the interface roughness and defectiveness, and therefore the presence of low density zones where atom interdiffusion can preferentially occur [6]. From the relative areas of the contributions to the Mössbauer spectrum, the thickness of the CoFe interfacial layer was evaluated to be $\sim 1.4 \mathrm{~nm}$, a value that remains practically unaffected by the increase of the thickness of the elemental layers. 
The XRD spectrum of the $100-5[\mathrm{Co}(3) / \mathrm{Fe}(9)]$ shows beside the reflection due to the substrate, the presence of a small (200) reflection which corresponds to the bcc $\mathrm{Fe}(200)$ and bcc $\mathrm{FeCo}(200)$ reflections $[5,8]$. By increasing the growing temperature up to $200^{\circ} \mathrm{C}$, figure $2(\mathrm{~b})$, the full width at half maximum of the (200) reflection slightly decreases while its intensity increases, suggesting an improvement of the sample crystallinity and an increase of the grain size. No other diffraction peaks were observed in the spectrum indicating the absence of any additional phases and crystal orientations, and therefore testifying the presence of a preferred [100] texture of the multilayer [5, 8]. The satellite peaks often observed around the main reflection in multilayered structures are absent probably due to the very low thickness of the multilayers which can hinder the establishing of a good structural coherence among the layers in particular on going toward the sample surface.

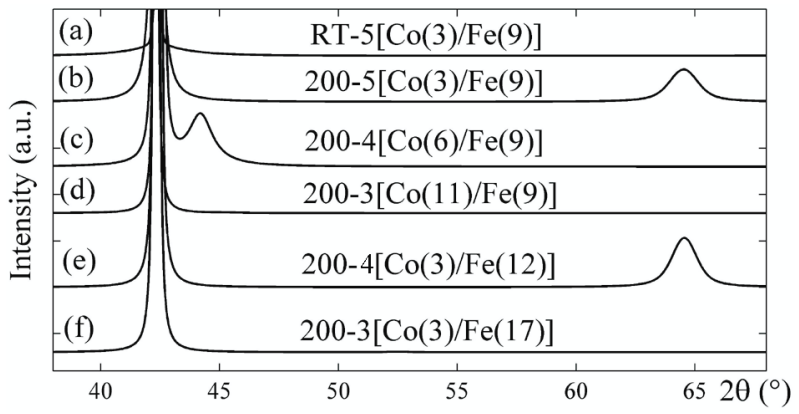

Figure 2 XRD pattern for the Fe/Co multilayers.

The AFM measurements show for the $\mathrm{RT}-5[\mathrm{Co}(3) / \mathrm{Fe}(9)]$ multilayer, figure $3(\mathrm{a})$, a morphology constituted by low-interconnected and irregular islands having a mean size of $195 \mathrm{~nm}$. From the analysis of the corresponding line profiles the mean height of the islands has been evaluated to be $\sim 3 \mathrm{~nm}$, which corresponds to the thickness of the Co capping layer. Under this nanostructured layer, the $\mathrm{Fe} / \mathrm{Co}$ multilayer grew as a continuous film. The increase of the growing temperature up to $200^{\circ} \mathrm{C}$, figure $3(\mathrm{~b})$, determines the Co-island coalescence, which gives rise to a more continuous morphology with the decrease of the mean surface roughness from 0.5 to $0.3 \mathrm{~nm}$.

The XRD spectra indicate that the increase of the Co layer thickness is detrimental for the [100] texture both for the multilayers grown at room temperature and up to $200^{\circ} \mathrm{C}$. The 200 $4[\mathrm{Co}(6) / \mathrm{Fe}(9)]$, figure 2(c), shows an appreciable [011] crystallographic texture which can be attributed to both bcc $\mathrm{Fe}(011)$ and bcc $\mathrm{FeCo}(011)[5,8,10]$. This reflection disappears by further increasing the Co layer thickness up to $11 \mathrm{~nm}$ [figure 2(d)]. The corresponding AFM images, figures 3(c) and 3(d), indicate that the increase of the Co layer thickness determines the formation of small grains on the top of a continuous base. The mean surface roughness decreases reaching a value of $0.06 \mathrm{~nm}$.

No reflections can be observed in the XRD patterns for the multilayers grown both at room temperature and at intermediate temperatures, increasing the Fe layer thickness. Only for the growing temperature of $200^{\circ} \mathrm{C}$, the increase of the Fe layer thickness up to $12 \mathrm{~nm}, 200$ $4[\mathrm{Co}(3) / \mathrm{Fe}(12)]$, favours both the [100] texture and an improvement of the sample crystallinity, figure 2(e). Compared with the $200-5[\mathrm{Co}(3) / \mathrm{Fe}(9)]$ multilayer, the AFM image [figure 3(e)] shows a decrease of both the island interconnection and the grain size. In effect, the peak-tovalley height $(\sim 8 \mathrm{~nm})$ evaluated from the line profiles suggests that the system is constituted by islands ( $100 \mathrm{~nm}$ wide) showing a snake-like morphology grown onto a continuous base. A further increase of the Fe layer thickness up to $17 \mathrm{~nm}, 200-3[\mathrm{Co}(3) / \mathrm{Fe}(17)]$, induces the coalescence of the islands into a continuous multilayered structure [figure 3(f)] capped by the Co layer having the more external part constituted by nano-islands. 

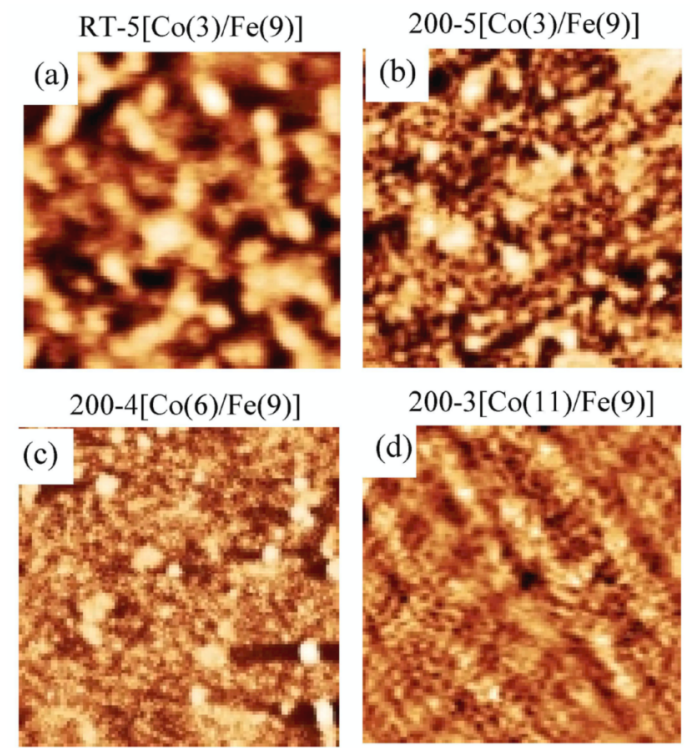

$200-3[\mathrm{Co}(11) / \mathrm{Fe}(9)]$

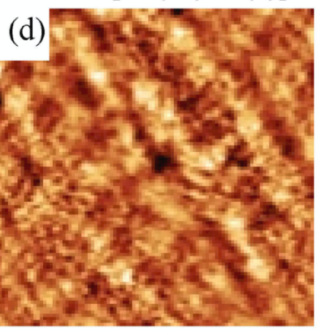

$200-4[\mathrm{Co}(3) / \mathrm{Fe}(12)]$

$200-3[\mathrm{Co}(3) / \mathrm{Fe}(17)]$
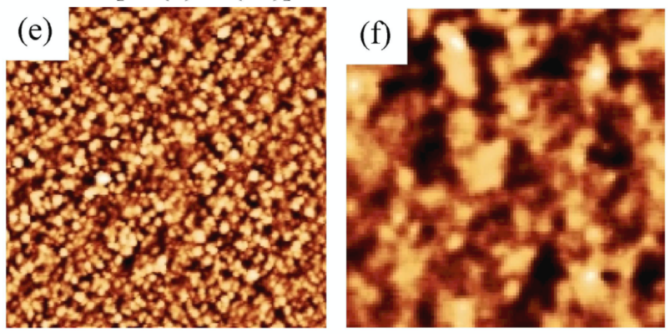

Figure 3 AFM images $\left(2 \times 2 \mu \mathrm{m}^{2}\right)$ for the Fe/Co multilayers.

The Z-contrast image of the 200-3[Co(11)/Fe(9)] sample obtained with STEM-HAADF is shown in figure 4(a). Grain morphology is clearly visible in the uppermost part of the structure with a lateral size of about $20 \mathrm{~nm}$, which is consistent with the results found by AFM [figure 3(d)]. Regular composition modulation of the Co and Fe layers can be observed in the vicinity of the substrate while, at a thickness of approximately $30 \mathrm{~nm}$, the growth front starts to rough resulting in interfacial intermixing between adjacent $\mathrm{Co}$ and $\mathrm{Fe}$ layers. These results are confirmed by EDS-STEM elemental map shown in figure 4(b). On the other hand, no significant intermixing effects were found in the $200-3[\mathrm{Co}(3) / \mathrm{Fe}(17)]$ sample. It is worth noting that in the investigated multilayers the layer thicknesses are in good agreement with the nominal values.
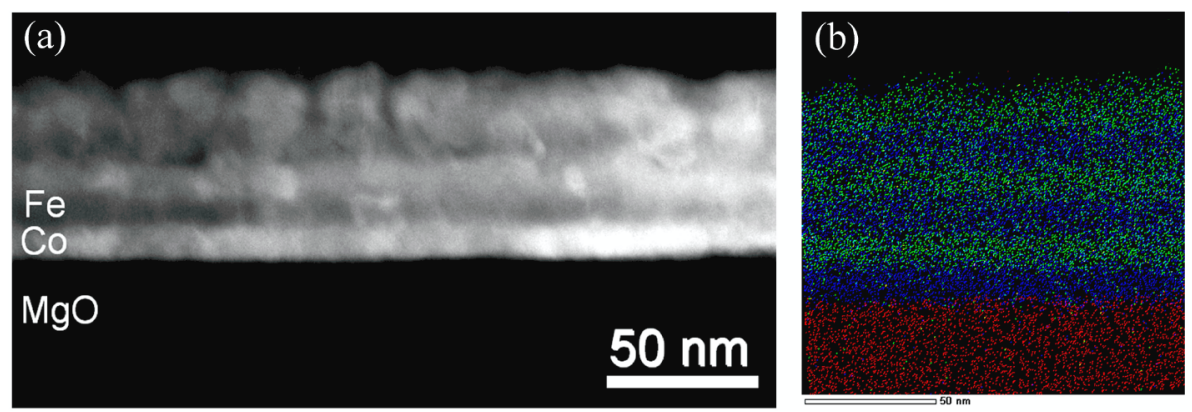

Figure 4 (a) HAADF cross section image of the 200-3[Co(11)/Fe(9)] sample; (b) EDS map for $\mathrm{Mg}$ (red), Co (blu) and Fe (green) taken in the same region. 
High resolution transmission electron microscopy of the $200-3[\mathrm{Co}(11) / \mathrm{Fe}(9)]$ multilayer shows that $\mathrm{Co}$ and $\mathrm{Fe}$ layers grew epitaxially on the $\mathrm{MgO}$ substrate with the fcc $\mathrm{Co}$ and bcc $\mathrm{Fe}$ structure, respectively. As an example, the cross section HREM image of the sample is reported in figure 5(a), showing two adjacent $\mathrm{Fe} / \mathrm{Co}$ grains in the immediate proximity of the substrate, separated by a grain boundary (GB).

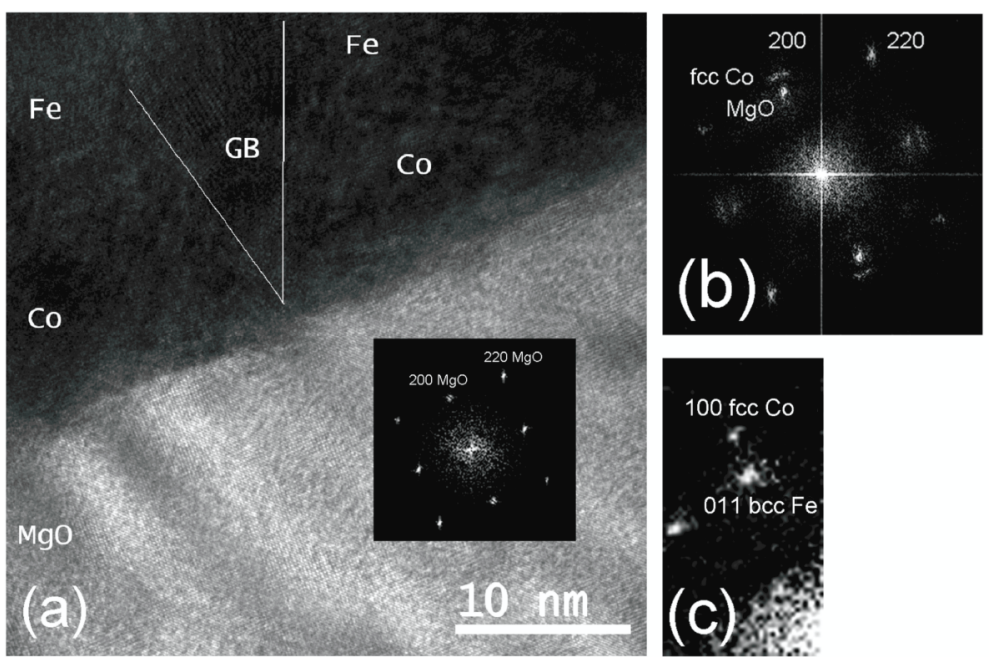

Figure 5 (a) (010) cross-sectional HREM of sample 200-3[Co(11)/Fe(9)] with the FFT pattern of the $\mathrm{MgO}$ substrate in the inset; (b) FFT pattern of the $\mathrm{Co} / \mathrm{MgO}$ interface region and (c) portion of the FFT pattern taken at the first Fe/Co interface.

As shown by the Fast Fourier Transformation (FFT) patterns taken at the $\mathrm{Co} / \mathrm{MgO}$ interface [figure 5(b)] and at first $\mathrm{Fe} / \mathrm{Co}$ interface [figure 5(c)], the structural relationship is $[011] \mathrm{Fe}_{\mathrm{bcc}}\left\|[100] \mathrm{Co}_{\text {fcc }}\right\|[100] \mathrm{MgO}$, even if a few degree tilt of the $[011] \mathrm{Fe}_{\text {bcc }}$ and $[100] \mathrm{Co}_{\text {fcc }}$ around the [100] of the $\mathrm{MgO}$ was found. By approaching the surface, a progressive loss of defined orientation relationship is observed, in particular for bcc Fe grains. As a result, the surface layer is characterized by bcc $\mathrm{Fe}(\mathrm{Co})$ differently oriented grains, with a preferential [011] texture axis, as shown in figure 6.

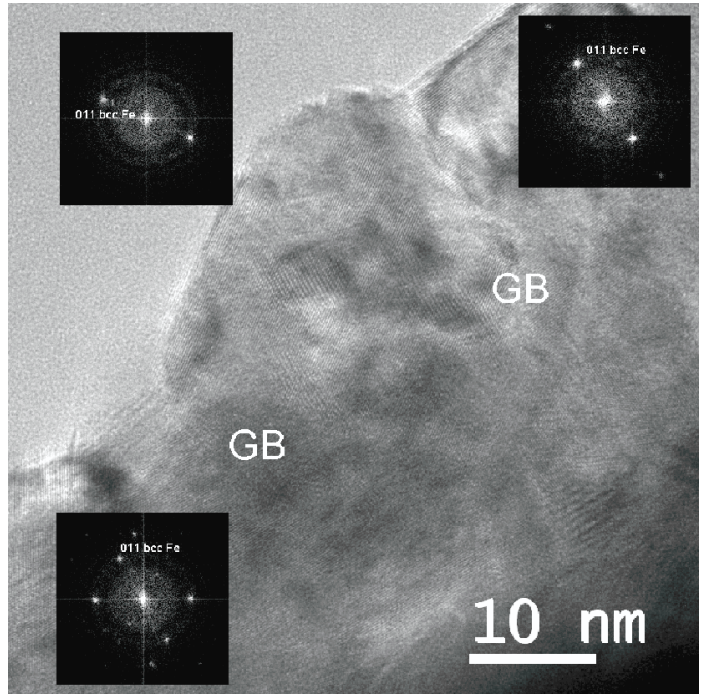

Figure 6 (010) cross-sectional HREM of the multilayer surface showing three different bcc $\mathrm{Fe}(\mathrm{Co})$ grains separated by grain boundaries. The FFT patterns of the differently oriented grains are shown in the insets. 
The gradual decrease of the orientation relationship of the Co and Fe layers going from the substrate towards the surface, together with the tilt of the grains along the [100] of the $\mathrm{MgO}$, account for the absence of XRD reflections in this sample. On the other hand, in the sample with the lowest Co layer thickness, 200-3[ $\mathrm{Co}(3) / \mathrm{Fe}(17)]$, the [011] texture of bcc Fe is almost absent and the contribution of Co is not sufficient to give detectable spots in the diffraction or FFT patterns, in agreement with the XRD results.

\section{Magnetic Properties}

The angular dependence of the normalized remanence $M_{r} / M_{s}$ and the $\Delta M$-plot for the $\mathrm{Fe} / \mathrm{Co}$ multilayers are reported in figure 7. The magnetic behaviour of the $\mathrm{RT}-5[\mathrm{Co}(3) / \mathrm{Fe}(9)]$ is characterized by a sine-like polar plot indicating the presence of an in-plane uniaxial anisotropy, figure 7(a). The corresponding $\Delta M$ curve reported in figure 7(b), except for a small positive contribution, is almost equal to zero for any applied field, indicating that the multilayer behaves as a non-interacting assembly of particles.

For the multilayers grown at room temperature, the increase of the Co layer thickness determines only a slight decrease of the anisotropy dispersion and therefore a slight increase of the in-plane anisotropy. On the contrary the increase of the Fe layer thickness induces an increase of the anisotropy dispersion allowing the establishing of a more isotropic magnetic behaviour.
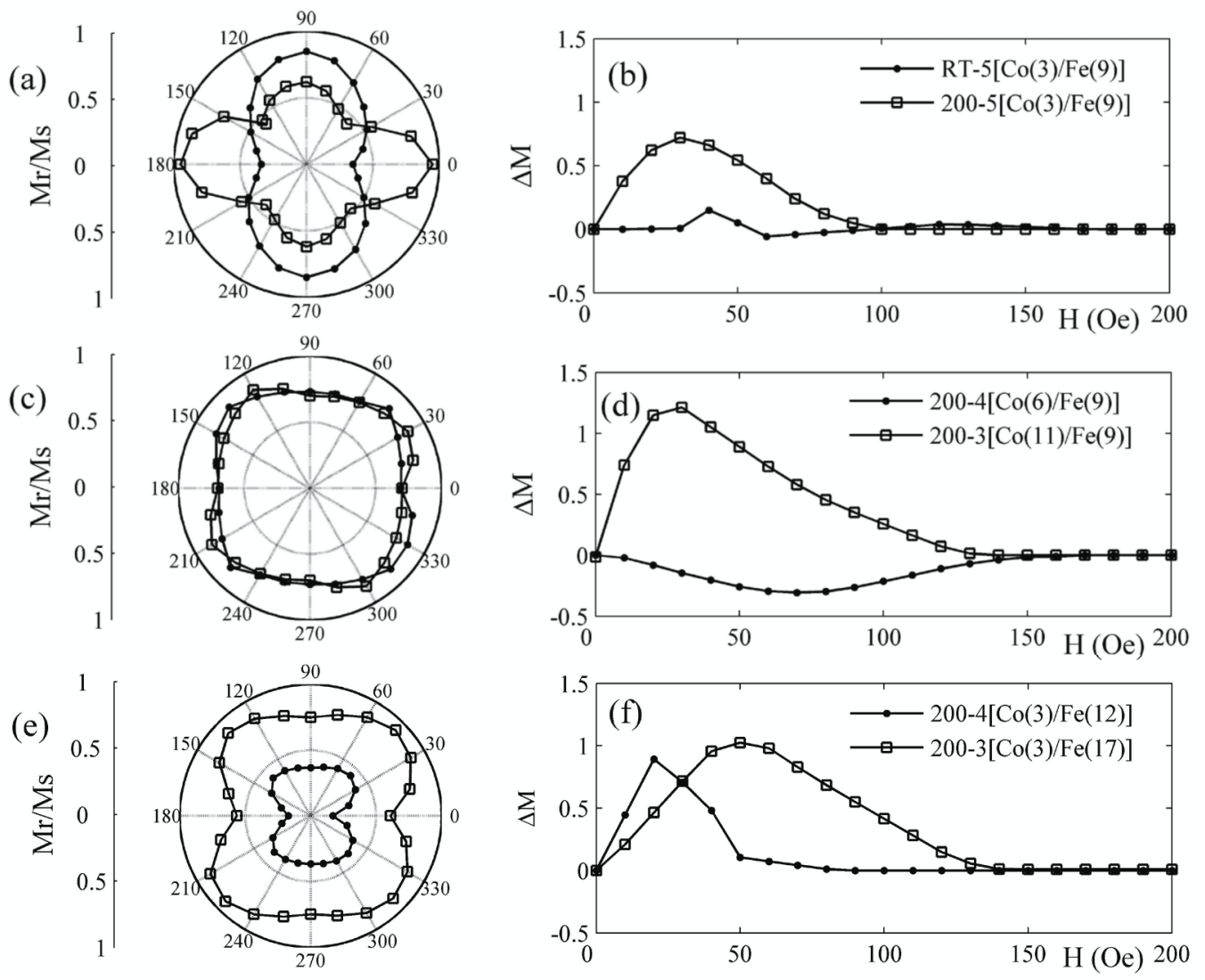

Figure 7 The $M_{r} / M_{s}$ polar plots (left-hand panel) and the $\Delta M$-plots (right-hand panel) for (a) (b) RT-5[Co(3)/Fe(9)] and 200-5[Co(3)/Fe(9)], (c) - (d) $200-4[\mathrm{Co}(6) / \mathrm{Fe}(9)]$ and 200$3[\mathrm{Co}(11) / \mathrm{Fe}(9)]$ and (e) - (f) $200-4[\mathrm{Co}(3) / \mathrm{Fe}(12)]$ and $200-3[\mathrm{Co}(3) / \mathrm{Fe}(17)]$ multilayers. For sake of clarity, the polar plots in (a) have been rotated of $90^{\circ}$ each other. 
By increasing the growing temperature, the coercivity remains practically unchanged while the normalized remanence evaluated along the easy axis monotonically increases (figure 8 for the $5[\mathrm{Co}(3) / \mathrm{Fe}(9)]$ multilayers). On the contrary along the hard direction the normalized remanence remains unchanged up to growing temperatures of $\sim 60^{\circ} \mathrm{C}$, upon which it slightly increases indicating the establishing of a small perpendicular contribution to the magnetization vector.

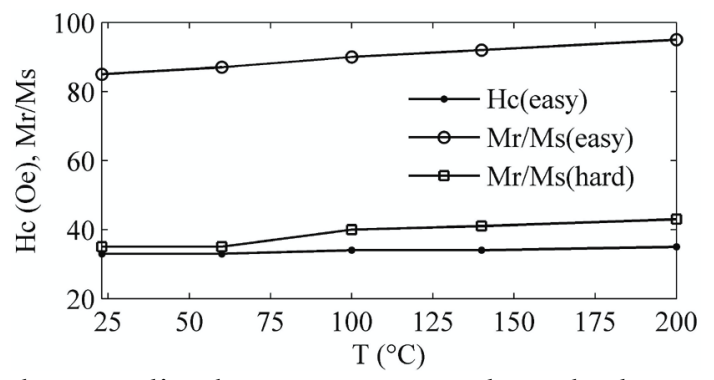

Figure 8 Coercivity and normalized remanence evaluated along the easy and hard axis directions as a function of growing temperature for the $5[\mathrm{Co}(3) / \mathrm{Fe}(9)]$ multilayers.

Moreover, a second preferred in-plane orientation of the easy magnetization axis establishes, and strengthens, giving rise, for the multilayer grown at $200^{\circ} \mathrm{C}$, to a magnetic behaviour having a four-fold symmetry [figure 7(a)]. Along the hard direction, i.e. at $45^{\circ}$ with respect to both the easy directions, the in-plane hysteresis loop shows a "transcritical shape", figure 9(a), which is typical of systems showing an appreciable out-of-plane component of the magnetization vector. The loop is due to the superposition of two contributions: (i) an abrupt transition at low applied fields due to wall displacement and (ii) an almost linear approach to the saturation for high applied fields, due to the progressive rotation of the magnetic moments [11]. The positive values of the $\Delta M$ curve in figure 7(b) indicate that the high-temperature growth favours the establishing among the layers of a significant exchange-coupling interaction.
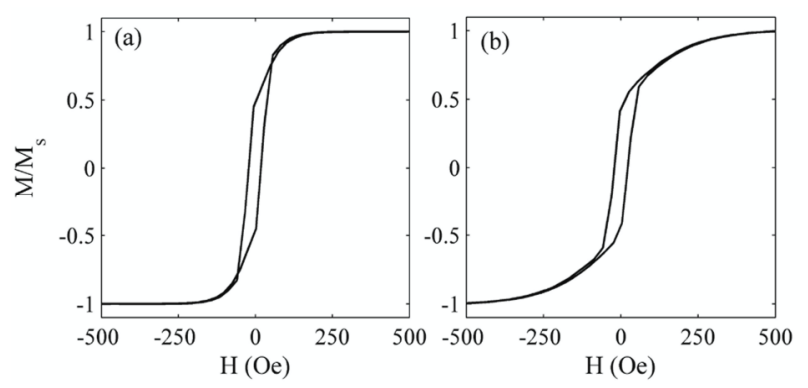

Figure 9 Longitudinal hysteresis loops for (a) $200-5[\mathrm{Co}(3) / \mathrm{Fe}(9)]$ measured with the field applied along the in-plane hard direction and (b) 200-4[Co(3)/Fe(12)] multilayer.

By increasing the Co layer thickness up to $6 \mathrm{~nm}, 200-4[\mathrm{Co}(6) / \mathrm{Fe}(9)]$, the two in-plane preferred orientations of the easy magnetization axis become comparable in strength, giving rise to a more in-plane isotropic behaviour as shown in figure 7(c). This may well be linked to the establishing of the [011] crystallographic texture, figure 2(c). The $\Delta M$ values become negative indicating the presence of demagnetizing interactions, figure 7(d). This change of the $\Delta M$ sign cannot be ascribed to a lower interlayer exchange-coupling interaction due for example to the presence of second phases into the multilayers or to the interfacial FeCo regions, since:

(i) no appreciable difference can be detected between the Mössbauer spectrum of the 200$4[\mathrm{Co}(6) / \mathrm{Fe}(9)]$ and that of the $200-5[\mathrm{Co}(3) / \mathrm{Fe}(9)]$ having a lower Co layer thickness, and

(ii) the shape of the MOKE loops suggests a behaviour typical of a single-layer magnet. 
On the contrary, it seems reasonable to assume that, among the small grains that form in the uppermost part of the multilayer [figures 3(c) and 4(a)], magnetostatic interactions can establish, in this way reducing the effectiveness of the interlayer exchange-coupling interactions.

A further increase of the Co layer thickness up to $11 \mathrm{~nm}$ leaves unchanged the magnetic behaviour, but determines the establishing of exchange-coupling interactions among both grains and layers, figure 7(d), reasonably because of a higher grain-packing fraction that gives raise to a smoother sample surface, figure 3(d).

The coercivity for the $\mathrm{Fe} / \mathrm{Co}$ multilayers grown at $200^{\circ} \mathrm{C}$, figure 10 , increases as a function of the Co layer thickness and reaches a value of $\sim 80$ Oe for the $200-3[\mathrm{Co}(11) / \mathrm{Fe}(9)]$ multilayer.

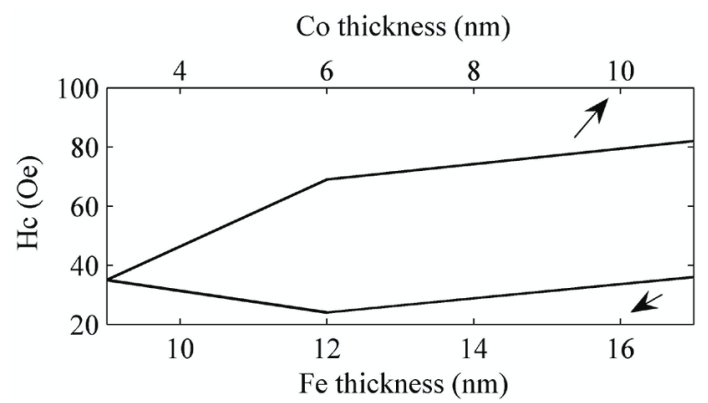

Figure 10 Behaviour of the coercivity as a function of the Co and Fe thickness for the Fe/Co multilayers grown at $200^{\circ} \mathrm{C}$.

Contrary to $\mathrm{Co}$, the increase of the Fe layer thickness is unable to change the sign of the $\Delta M$ curves and only favours a strengthening of the exchange-coupling interaction in the multilayers, figure 7(f). Moreover, for the $200-4[\mathrm{Co}(3) / \mathrm{Fe}(12)]$ a narrowing of the $\Delta M$-curve width occurs, probably due to the formation of a snake-like morphology, figure 3(e). The corresponding remanence curve, figure 7(e) full points, indicates the establishing of (i) an in-plane isotropic behaviour, and (ii) a significant out-of-plane contribution to the magnetization vector at the origin of both the very low values of the $M_{r} / M_{s}$ curve along any in-plane direction and the transcritical shape of the hysteresis loop [figure 9(b)]. This out-of-plane magnetization component determines the appearance of a magnetic morphology constituted by irregular stripelike domains, figure 11(a).
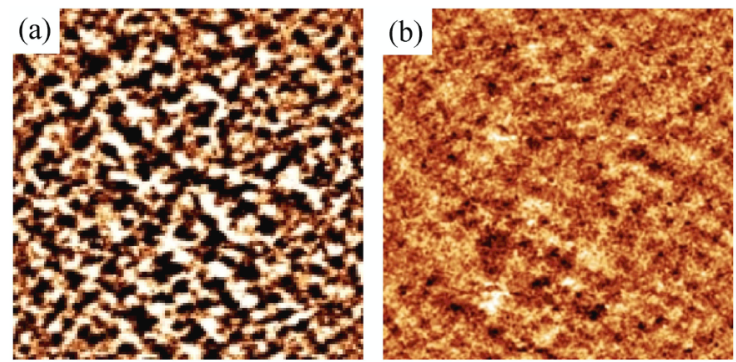

Figure 11 MFM images $\left(2 \times 2 \mu \mathrm{m}^{2}\right)$ for (a) $200-4[\mathrm{Co}(3) / \mathrm{Fe}(12)]$ and (b) $200-3[\mathrm{Co}(3) / \mathrm{Fe}(17)]$.

By further increasing the Fe layer thickness, the out-of-plane magnetization component decreases in strength, and completely disappears for the $200-3[\mathrm{Co}(3) / \mathrm{Fe}(17)]$, as suggested by the higher values of the corresponding $M_{r} / M_{s}$ curve, figure 7(e) open circles, and by the absence of any magnetic morphology, figure 11(b).

The coercivity as a function of the Fe layer thickness, reported in figure 10, initially decreases reaching a minimum for $t_{F e}=12 \mathrm{~nm}$ and then slightly increases. This trend can be explained considering that the $\mathrm{Fe} / \mathrm{Co}$ multilayers can be treated as exchange-coupled spring 
magnets, i.e. systems constituted by soft $(\mathrm{Fe})$ and hard $(\mathrm{Co})$ magnetic phases exchange coupled at the interfaces. The increase of the soft Fe layer thickness gives rise to the initial softening of the magnetic behaviour of the multilayer. When the thickness of the soft layer becomes much higher than that of the hard one the structural disorder increases favouring an increase of the preferential sites for the domain wall pinning [12].

\section{Conclusions}

$\mathrm{Fe} / \mathrm{Co}$ multilayers were grown at different temperatures onto $\mathrm{MgO}-(100)$ monocrystalline substrates, changing the Co and Fe layer thickness.

By growing the multilayers at room temperature, a thin interfacial region forms between the elemental layers constituted by $\mathrm{FeCo}$ and $\mathrm{Co}(\mathrm{Fe})_{\mathrm{ss}}$. The magnetic behaviour is typical of a system showing an in-plane uniaxial magnetocrystalline anisotropy. The increase of the elemental layer thickness induces a slight decrease (in the case of Co) or increase (in the case of $\mathrm{Fe}$ ) of the anisotropy dispersion along the easy magnetization direction.

The increase of the growing temperatures determines a lower diffusion at the interfaces with the gradual disappearance of the $\mathrm{Co}(\mathrm{Fe})_{\mathrm{ss}}$ and the formation of the only $\mathrm{FeCo}$ compound in the multilayers grown at $200^{\circ} \mathrm{C}$. For this temperature, the samples are characterized by (i) regularly modulated layers of $\mathrm{Fe}$ and $\mathrm{Co}$, which start to rough at a depth of $\sim 30 \mathrm{~nm}$ from the surface, (ii) a [100] crystallographic texture, and (iii) two in-plane preferred orientations of the easy magnetization axis.

The increase of the Co layer thickness determines the establishing of an isotropic in-plane magnetic behaviour and a preferred [011] crystallographic texture with a structural relationship $[011] \mathrm{Fe}_{\text {bcc }}\left\|[100] \mathrm{Co}_{\text {fcc }}\right\|[100] \mathrm{MgO}$. Depending on the Co layer thickness, the intergrain interactions favour alternatively the magnetized or demagnetized state of the multilayer.

The increase of the $\mathrm{Fe}$ layer thickness up to $12 \mathrm{~nm}$, for growing temperatures of $200^{\circ} \mathrm{C}$, determines (i) an improvement of the [100] texture of the $\mathrm{Fe} / \mathrm{Co}$ multilayers and (ii) an appreciable out-of-plane component of the magnetization vector which gives rise to a magnetic morphology constituted by stripe-like domains. For Fe layer thickness higher than $12 \mathrm{~nm}$, the magnetization vector preferentially lies into the film plane and the structural relationship $[011] \mathrm{Fe}_{\mathrm{bcc}}\left\|[100] \mathrm{Co}_{\text {fcc }}\right\|[100] \mathrm{MgO}$ establishes as in the case of the multilayers with the Co layer thickness higher than $3 \mathrm{~nm}$.

\section{References}

[1] Ciprian R and Carbucicchio M 2010 J. Phys.: Conf. Ser. 200, 072018(4pp)

[2] Aurongzeb D, Ram K B and Menon L 2005 Appl. Phys. Lett. 87, 172509(3pp)

[3] Park M H, Hong Y K, Gee S H, Mottern M L, and Jang T W 2002 J. Appl. Phys. 91, 721820

[4] Kalska B, Blomquist P, Häggström L, and Wäppling R 2001 J. Phys.: Condens. Matter 13, 2963-70

[5] Gupta R, Khandelwal A, Ansari R, Gupta A, and Nair K G M 2009 Surf. Coat. Technol. 203, 2717-20

[6] Carbucicchio M and Ciprian R 2010 IEEE Trans. Magn. 46, 397-400

[7] Cooke M D, Wang L-C, Watts R, Zuberek R, Heydon G, Rainforth W M and Gehring G A 2000 J. Phys. D: Appl. Phys. 33, 1450-9

[8] Shikada K, Ohtake M, Kirino F and Futamoto M 2009 J. Appl. Phys. 105, 07C303(3pp)

[9] Jergel M, Cheshko I, Halahovets Y, Siffalovic P, Mat'ko I, Senderak R, Protsenko S, Majkova E and Luby S 2009 J. Phys. D: Appl. Phys. 42, 135406(8pp)

[10] Fu Y, Miyao T, Cao J W, Yang Z, Matsumoto M, Liu X X and Morisako A 2007 J. Magn. Magn. Mater. 308, 165-9 
[11] Ausanio G, Iannotti V, Lanotte L, Carbucicchio M and Rateo M 2001 J. Magn. Magn. Mater. 226/230, 1740-2

[12] Carbucicchio M, Bennett S, Berry F J, Prezioso M, Rateo M and Turilli G $2003 \mathrm{~J}$. Appl. Phys. 93, 7631-7633 